Ortiz Martínez, E., Santos Jaén, J.M. y Marín Hernández, S. (2020). Evaluación continua en la enseñanza universitaria de la contabilidad. Revista de Investigación Educativa, 38(1), 109-129.

DOI: http://dx.doi.org/10.6018/rie.329781

\title{
Evaluación continua en la enseñanza universitaria de la contabilidad
}

\section{Continuous assessment at university teaching of accounting}

\author{
Esther Ortiz Martínez, José Manuel Santos Jaén y Salvador Marín Hernández \\ Departamento Economía Financiera y Contabilidad, Universidad de Murcia (España)
}

\begin{abstract}
Resumen
En España el uso de la evaluación continua en las notas universitarias es consecuencia de nuestra adaptación al EEES y por lo tanto aún está por determinar su efecto en el aprendizaje del alumno. Por ello nos planteamos en este estudio, si la evaluación continua aumenta las garantías de éxito en el examen final, y si el número de pruebas intermedias que se realizan y su formato, afecta a este aprendizaje. Se ha analizado una muestra de 1670 notas de distintas asignaturas universitarias de Contabilidad Financiera durante los últimos tres cursos académicos. Los resultados obtenidos permiten aceptar las hipótesis de partida de que efectivamente la evaluación continua afecta de forma positiva a la nota del examen final. Asimismo, también el tipo de prueba parcial influye en esta nota, y no el número de pruebas de evaluación continua realizadas. Se confirma así, como en estudios previos, que existe un efecto positivo de la evaluación continua sobre el rendimiento académico. No obstante, es importante un buen diseño de estas pruebas, pues aunque también los antecedentes abogan por modificarlas de un curso a otro, pueden determinar la nota del examen final.

Palabras clave: evaluación continua; universidad; educación superior; proceso de aprendizaje.
\end{abstract}

Correspondencia: Esther Ortiz Martínez, esther@um.es. Univesidad de Murcia Facultad de Economía y Empresa, Campus de Espinardo, 30100-Murcia. 


\begin{abstract}
Continuous assessment has been used in Spanish universities after the implementation of the European Higher Education Area (EHEA), and so it is important to study its effects on students' acquisition of knowledge. The goal of this paper is to analyse whether continuous assessment shows a positive effect university students' learning, which is reflected in final exam scores. It also sets out to prove whether the number of mid-point tests and their different formats affects the students' learning process. We have analysed a sample of 1670 scores from different subjects of Financial Accounting for the last three academic years. The results allow us to confirm our initial hypothesis that continuous assessment positively affects final exam scores, and so it has a positive impact on the learning process. The type of tests taken as part of the students' continuous assessment likewise affects their learning process; however, it is important that mid-point tests are designed carefully. Even though previous studies on the matter have pointed towards the benefits of switching the format of mid-point tests every year, their design can still determine the students' final exam scores.

Keywords: continuous assessment; university; higher education; learning process.
\end{abstract}

\title{
Introducción
}

La implantación del Espacio Europeo de Educación Superior (EEES) en las aulas universitarias españolas ha conllevado una serie de cambios en la docencia, entre los que nos centramos en este trabajo en la evaluación continua. De manera que, pasando de un sistema de evaluación en el que la nota de la asignatura se correspondía con la de un examen final, se instaura un proceso de evaluación continua durante el curso, como evaluación formativa, que pretende mejorar el proceso de aprendizaje del alumno y, por lo tanto, tener una repercusión positiva en el examen final, y así en la calificación obtenida en la asignatura. El aprendizaje a través de competencias se introduce en la universidad por el EEES, con mayor contenido pedagógico (Navaridas Nalda, Jiménez Trends y Fernández Ortiz, 2016), y ello conlleva también cambios en las modalidades de enseñanza y aprendizaje, y por lo tanto en la evaluación (Moreno-Murcia, Aracil y Reina, 2014). La implantación de la evaluación orientada al aprendizaje en distintos países ha tenido consecuencias diversas (Birenbaum et al., 2015; Pereira, Niklasson y Flores, 2017) y en España también ha supuesto otra orientación distinta a la tradicional.

Este proceso de aprendizaje durante el curso, en la práctica conlleva toda una serie de incertidumbres, sobre las que tratamos de obtener algún tipo de resultado en este trabajo, ya que no existen antecedentes claros sobre el efecto real de la evaluación continua en los resultados académicos de los alumnos, a lo que se suma que también el diseño de la evaluación continua puede estar determinando sus resultados, tanto el número de pruebas de las que se compone, como el formato utilizado en las mismas. 


\section{Antecedentes}

A partir de los años ochenta con la implantación del EEES, el concepto de evaluación ha evolucionado desde la evaluación tradicional en el aula universitaria: el examen final, hasta el proceso de evaluación continua en el que se prima el aprendizaje continuo (Fernández, 2017; Palacios Picos, 2004). Tradicionalmente "evaluar" era poner una nota final al alumno, pero no una tarea (Trigueros Cervantes, Rivera García y De la Torre Navarro, 2012): “cuyo objetivo es la valoración del proceso de enseñanza y aprendizaje llevado a cabo" (p. 480). Los métodos de enseñanza-aprendizaje en la universidad española tienen efecto sobre el rendimiento académico medido a través de la nota media (Coll-Serrano, Pardo-García y Pérez, 2018).

En la docencia, el proceso de evaluación es casi lo más importante que el profesor hace, pues debe plasmar en una calificación el grado de consecución de unos objetivos de aprendizaje (Trotter, 2006) y se debe configurar como un proceso (Alcáraz, Fernández y Sola, 2012) en el que se trata de enseñar, más que medir (Carless, 2007). El profesor "excelente" también se va a preocupar de que el método de evaluación contribuya a su aprendizaje (Escámez Sánchez, 2016).

El método de evaluación puede condicionar sobremanera al alumno, pues dependerá de él cómo enfoca su estudio o dedicación a la asignatura en cuestión (Crooks, 1988). Existen antecedentes previos que demuestran que el estudiante se entrena para un tipo de prueba de evaluación, con independencia de si domina o no la materia (Gibbs y Simpson, 2004). El formato y tipo de evaluación es un factor contextual que va a determinar el aprendizaje, y por lo tanto la expresión del mismo que se concreta en una nota (Monroy y Hernández, 2014).

Anteriormente al realizar un único examen final no se aplicaba el sentido formativo de la evaluación, pues no se cumplía con la característica principal de la evaluación formativa: contribuir al aprendizaje del alumno a través del suministro de información sobre cómo va progresando (Yorke, 2003) y, por lo tanto, el alumno tendía a dejar el estudio para demasiado tarde, con los consiguientes malos resultados (Dowell et al., 2007, p. 4). Además de que el reducir las pruebas a una sola, implica que no se puede dar cabida a la gran diversidad de estudiantes que deben ser evaluados (Gibbs y Simpson, 2004) porque por ejemplo centrar la evaluación en una prueba escrita puede discriminar a estudiantes con pocas habilidades para la escritura o algún tipo de dislexia (Isaksson, 2008).

La realización de pruebas intermedias en una evaluación continua se configura como un "feedback" que se va dando al alumno, de manera que pueda ir enfocando mejor su aprendizaje, siendo este "feedback" un rasgo fundamental de una evaluación formativa (Black, 2015; Black y Willian, 1998; Carless, 2015; Flores, Veiga Simao, Barros y Pereira, 2015). Heitink, Van der Kleij, Veldkamp, Schildkamp y Kippers (2016) sostienen que, si la evaluación formativa no tiene el efecto deseable sobre el aprendizaje, es porque no está correctamente implementada, y para ello se plantean si tienen que existir unos requisitos previos con respecto al profesor, al estudiante, la evaluación y el contexto, de cara a esta implantación. Resaltan el papel crucial que juega el profesor entre los requisitos analizados a través de meta análisis de estudios previos sobre la materia.

En la Memoria del Grado en Administración y Dirección de Empresas (ADE) de la Universidad de Murcia para las asignaturas de Contabilidad Financiera, sobre las que 
vamos a realizar este estudio empírico, el porcentaje de la nota final que se cumplimenta a través de las pruebas parciales de evaluación continua es el 30\%.

El número y diseño de las pruebas de evaluación continua se va modificando de un curso académico a otro y se intentan diseñar cubriendo un amplio espectro de tipos de pruebas, tal y como es deseable para una evaluación exhaustiva (Crisp, 2007, p 579). Además de que también se incorporan las nuevas tecnologías para la realización de alguna de las pruebas de evaluación continua, tal y como recomiendan algunos autores (Gibbs, 2006; Gibbs y Simpson, 2004). La Estrategia Europea 2020 promueve la innovación en los centros de educación superior para crear entornos de aprendizaje más interactivos, lo que implica el uso de las posibilidades que nos brindan hoy en día las tecnologías de la información y las comunicaciones (Camacho Miñano, Urquía Grande, Pascual Ezama y Rivero Menéndez, 2016; Gámiz Sánchez y Gallego Arrufat, 2016; Martínez Clares, Pérez Cusó y Martínez Juárez, 2016).

Los antecedentes sobre la evaluación continua que han realizado estudios empíricos se han basado principalmente en la realización de encuestas y entrevistas, tanto a alumnos, como a profesores, como es el caso de Hernández (2012), que concluye que la evaluación continua sirve de apoyo y motivación al aprendizaje de los alumnos, pero para ello el docente debe dar el retorno, sobre las pruebas que se vayan realizando, en tiempo y forma. Fernández-Ponce, Arbulo Echevarría, Muñoz-Miranda, Aguado y García-Cozar (2017) también realizan entrevistas, basándose en datos cualitativos, para resaltar que, tanto para profesores como para alumnos, la actual evaluación continua les resulta positiva. Otra línea de estudios se basa en el análisis de las guías docentes de las asignaturas, para analizar los sistemas de evaluación, concluyendo que aún el examen final sigue siendo clave (San Martín Gutiérrez, Jiménez Torres y Jerónimo Sánchez-Beato, 2016). En esta línea, Castejón y Santos (2011) concluyen que en cuanto a la metodología participativa en clase y la utilización de sistemas de evaluación formativa, no concuerdan lo que se establece a priori con lo que luego se pone en práctica. Otros trabajos sí que analizan rendimientos académicos de los alumnos como es nuestro propósito, pero no en cuanto a evaluación continua, sino centrándose en cómo influye en ellos el aprendizaje cooperativo (Vallet-Bellmunt, Rivera-Torres y Vallet-Bellmunt, 2017). Rodríguez-Gómez, Quesada-Serra e Ibarra-Sáiz (2016) unen la entrevista como método cualitativo, al análisis cuantitativo de las notas, para resaltar el efecto positivo de la evaluación continua.

Existen trabajos que subrayan el efecto positivo de la evaluación formativa sobre el rendimiento académico, como el de Cano (2011), que estudia las notas obtenidas por más de 750 estudiantes durante siete cursos académicos, concluyendo que el uso de la evaluación continua aumenta el número de aprobados, así como también mejora las notas obtenidas. Ya que el "feedback" de estas técnicas de evaluación tiene efecto positivo en los rendimientos académicos (Parkes, 2010). El trabajo de Fraile, López, Castejón, y Romero (2013) también concluye que el rendimiento académico mejora cuando se usa la evaluación continua únicamente, o la evaluación mixta. Gil Flores y García Jiménez (2014) se centran en analizar cómo la evaluación formativa sí que influye en los resultados del aprendizaje, pero en su caso además incluyen una variable que mide el nivel socioeconómico del entorno de los alumnos, que obtienen que es incluso más determinante de los resultados alcanzados. Gargallo López et al. (2014) introducen 
el análisis de la evaluación continua a través de diferentes pruebas, como es el caso de este estudio, como herramienta de aprendizaje, comprobando que el efecto es positivo.

Un grupo de antecedentes se ha basado en la utilización del meta-análisis, para analizar las conclusiones de trabajos anteriores y ver el efecto de la evaluación formativa en los resultados del aprendizaje. Lo obtenido es dispar porque los estudios analizados son muy diversos entre sí (Black y William, 1998). Estos trabajos de metaanálisis, resaltan tanto el efecto positivo de la evaluación formativa (Fuchs y Fuchs, 1986; Kluger y DeNisi, 1996), como no pueden concluir al respecto (Bennet, 2011; Dunn y Mulvenon, 2009; Shute, 2008). También utilizando meta-análisis Barrientos Hernán y López Pastor (2017) aseguran que existen pocos estudios que se centran en la evaluación orientada al aprendizaje.

Para el caso concreto que nos ocupa, que se centra en la asignatura de Contabilidad Financiera, encontramos antecedentes como el de Sánchez-Martín, Pascual-Ezama, y Delgado-Jalón (2017), en el que sí que analizan resultados académicos de los alumnos, y además estudian el proceso de evaluación acumulativo previo a la calificación final, concluyendo que obtienen mejores resultados académicos. Pascual Ezama, Camacho Miñano, Urquía Grande y Müller (2011) también realizan un análisis empírico a partir de las notas de la asignatura de Contabilidad Financiera, y llegan a la conclusión de que se debería revisar este nuevo método de evaluación consecuencia del EEES, pues no permite a los estudiantes con mejores notas en el examen final obtener una mejor calificación si no lo han demostrado previamente en las pruebas intermedias. Jareño Cebrián y López García (2015) estudian los resultados de nueve asignaturas obligatorias en el Grado en Administración y Dirección de Empresas, entre las que se encuentran las de Análisis Contable y Contabilidad de Costes, y también concluyen que existe correlación positiva entre las calificaciones de la evaluación continua y la nota obtenida en el examen final, destacando que la nota final en la asignatura es mayor con este sistema.

En cambio, Poza-Lujan, Calafate, Posadas-Yague y Cano (2016) cuantifican el "sobrecoste" que supone para el docente la evaluación continua, y su efecto en la nota de los estudiantes, llegando a la conclusión de que utilizar este tipo de evaluación no tiene un impacto significativo en las notas. Crisp (2007) también estudia las notas y el efecto que parece tener sobre ellas la evaluación continua, y no puede concluir que el hecho de dar ese "feedback" a los alumnos mejore las calificaciones.

Aunque en general existen antecedentes sobre la materia que prueban el efecto positivo de la evaluación formativa sobre el rendimiento académico (Cano, 2011; Fraile et al., 2013; Isaksson, 2008; Jareño Cebrián y López García, 2015; Parkes, 2010; Sánchez-Martín et al., 2017); otros en cambio subrayan que no compensa la función coste-beneficio de aplicarla (Crisp, 2007; Pascual Ezama et al., 2011; Poza-Lujan et al., 2016), y no muchos de ellos utilizan datos reales con notas de alumnos universitarios con los que realizar un análisis sobre el que obtener resultados que puedan contribuir a la materia.

De ahí que en este trabajo nos planteemos, con una base de datos de 1670 notas de asignaturas de Contabilidad Financiera, si efectivamente el efecto de la evaluación continua sobre los resultados del alumno es positivo, pues se supone que mejora el proceso de aprendizaje, y si influye sobre este efecto el diseño de la evaluación continua, tomando como tal el número de pruebas intermedias realizadas y el formato de cada una de ellas. 


\section{Método}

\section{Objetivos}

Pretendemos estudiar los resultados académicos de los alumnos en distintas asignaturas de Contabilidad Financiera que se recogen en la nota del examen final, y cómo pueden estar influenciados por el proceso de evaluación continua, concretamente por el número de pruebas intermedias que constituyen esta evaluación continua, y el formato de las mismas.

\section{Hipótesis}

Las hipótesis a contrastar en este trabajo son las siguientes:

$\mathrm{H}_{1}$ : La evaluación continua afecta al aprendizaje y por lo tanto a los resultados obtenidos en la nota del examen final

$\mathrm{H}_{2}$ : El número de pruebas de evaluación continua afecta al aprendizaje y por lo tanto a los resultados obtenidos en la nota del examen final

$\mathrm{H}_{3}$ : El tipo de prueba de evaluación continua afecta al aprendizaje y por lo tanto a los resultados obtenidos en la nota del examen final

\section{Participantes}

Hemos elaborado una base de datos en la que incluimos las notas obtenidas en los tres últimos cursos académicos, es decir: 2014-2015, 2015-2016 y 2016-17, en las distintas asignaturas de Contabilidad Financiera en el Grado en Administración y Dirección de Empresas (ADE) y en el de Ciencias Políticas (CC Políticas) de la Universidad de Murcia. Lo que nos lleva a contar finalmente con una muestra de 1670 notas (las características de la muestra las recogemos en la Tabla 1).

Tabla 1

La muestra

\begin{tabular}{|c|c|c|c|c|c|c|c|c|c|}
\hline \multirow[t]{2}{*}{$\begin{array}{l}\text { Curso } \\
\text { académico }\end{array}$} & & \multicolumn{2}{|c|}{ Asignatura y cuatrimestre } & \multicolumn{2}{|c|}{ Convocatoria } & \multicolumn{2}{|c|}{ Sexo } & \multicolumn{2}{|c|}{$\begin{array}{l}\text { Número } \\
\text { de pruebas } \\
\text { parciales }\end{array}$} \\
\hline & $\begin{array}{l}\text { № } \\
\text { notas }\end{array}$ & & № notas & & $\begin{array}{l}\text { № } \\
\text { notas }\end{array}$ & & & & $\begin{array}{c}\text { № } \\
\text { notas }\end{array}$ \\
\hline \multirow[t]{2}{*}{ 2014-15 } & 454 & $\begin{array}{l}\text { Contabilidad Financiera I } \\
\text { (2344) ADE }\end{array}$ & 554 & 1 & 800 & Varones & 956 & 2 & 587 \\
\hline & & 1er cuatrimestre & & & & & & & \\
\hline
\end{tabular}




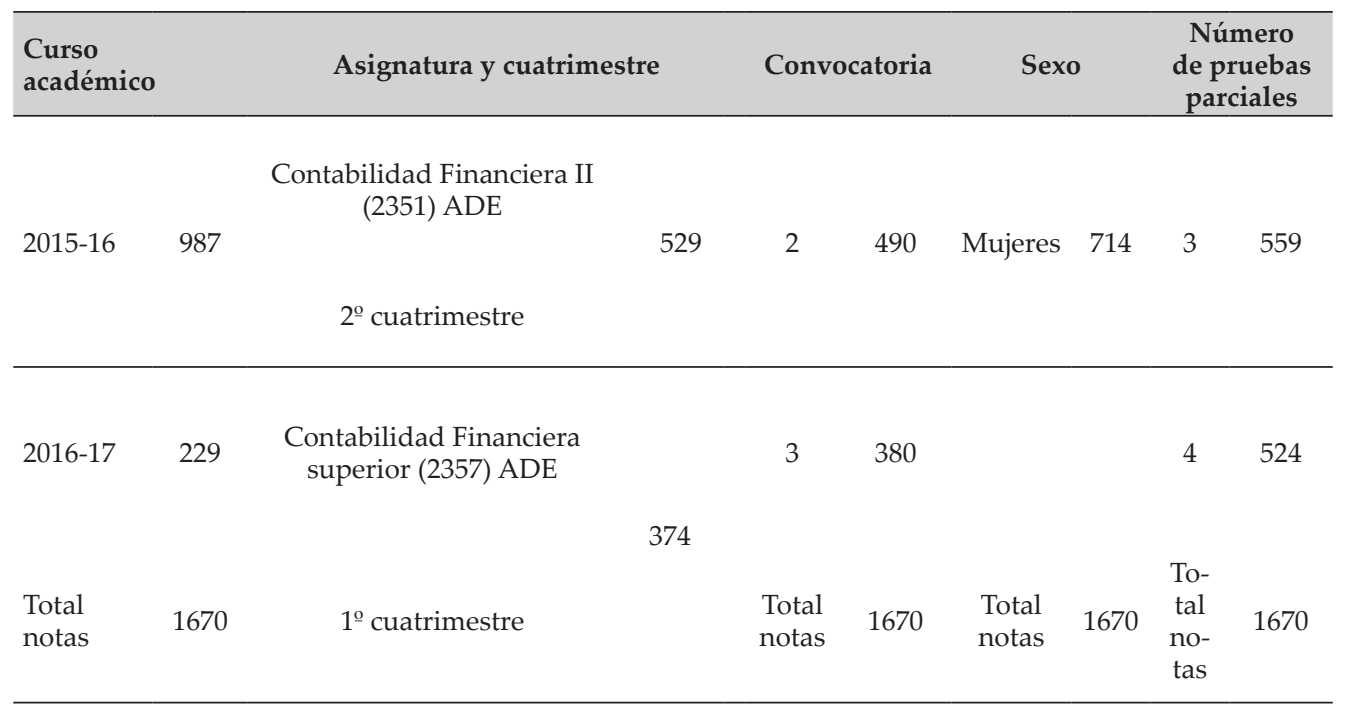

Introducción a la Contabilidad (2210) CC Políticas

$1^{\circ}$ cuatrimestre

\section{Instrumentos}

Dentro de la evaluación continua se realizan a los alumnos una serie de pruebas de evaluación intermedias, que a su vez cuentan con una calificación y suponen un porcentaje de la nota final. En nuestro caso supone el 30\% de la calificación final de la asignatura. A su vez, existe cierta flexibilidad a la hora de calificar este $30 \%$, introduciendo distintas pruebas intermedias en el cuatrimestre, de ahí que hayamos recogido tanto el dato del número de pruebas intermedias que se han valorado en cada curso académico, como su tipología, ya que las mismas han cambiado, al intentar el docente buscar una vía para que efectivamente la evaluación continua sea útil a la hora de conseguir el objetivo final de aprobar la asignatura y facilitar un aprendizaje continuo de la misma (Tabla 2). 
Tabla 2

Tipología de pruebas de evaluación continua y su ponderación por asignatura y curso académico

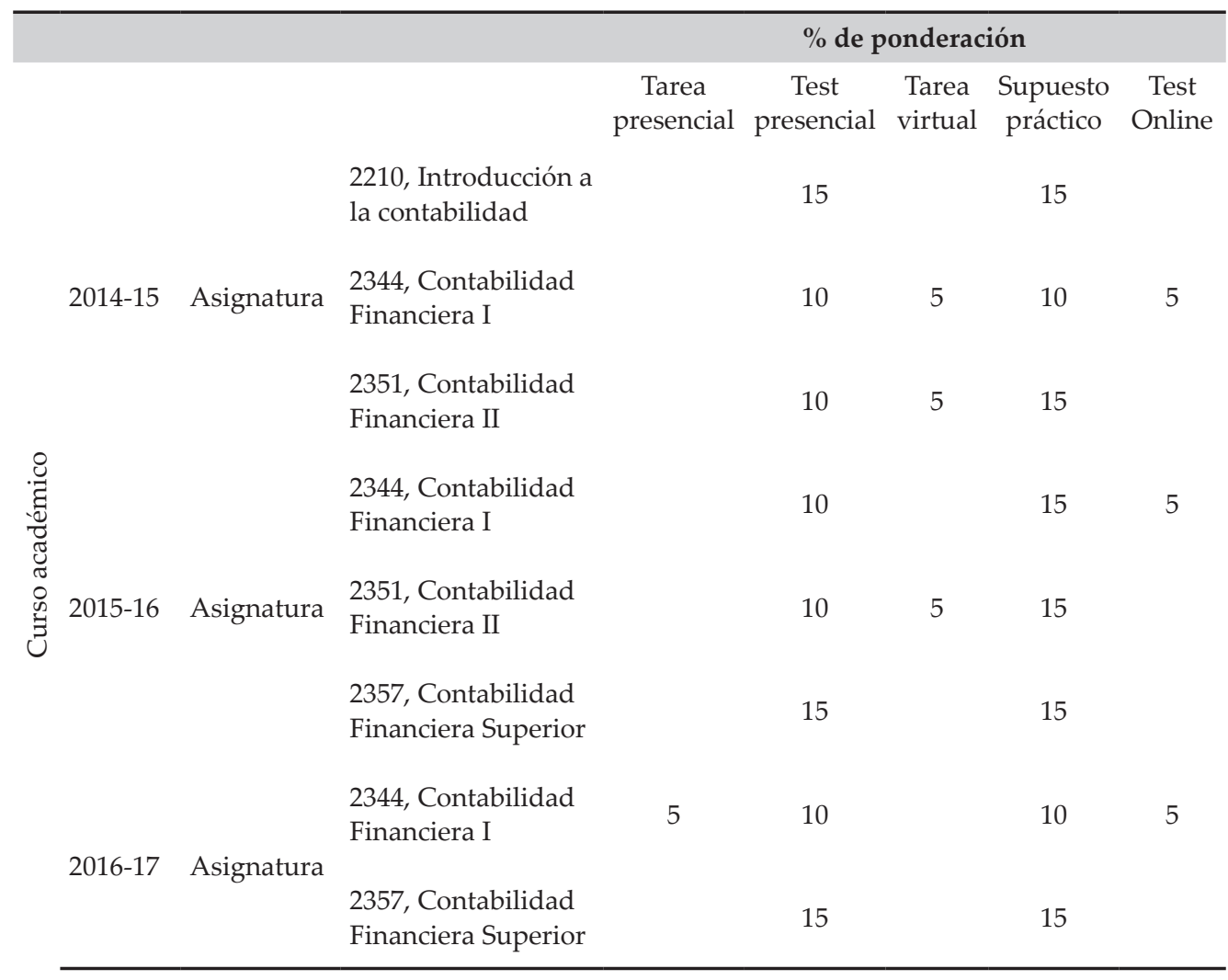

\section{Procedimiento y análisis de datos}

El análisis estadístico de los datos se ha realizado con el programa SPSS Versión 19 para Windows. Para las variables cualitativas, se ha obtenido el número de casos presentes en cada categoría y el porcentaje correspondiente; y para las variables cuantitativas, los valores mínimo, máximo, media y desviación típica, con los que se ha hecho un análisis inicial descriptivo.

Además, las variables cuantitativas (incluidas en la Tabla 1) se han recodificado en nuevas variables con el objeto de poderlas transformar en variables cualitativas. De manera que las calificaciones expresadas en la escala de 0 a 10, las hemos recodificado en dos variables cualitativas, según la nota sea menor a un cinco, es decir, un suspenso, o igual o mayor a cinco, un aprobado. Estas dos variables a su vez las hemos cruzado con otras variables cualitativas de la base de datos, realizando un análisis de tablas de contingencia y el contraste estadístico chi-cuadrado, considerando significatividad estadística de al menos el 95\%. 
En último lugar planteamos dos modelos de regresiones lineales con el objeto de poder contrastar nuestras hipótesis iniciales, y determinar en qué medida la nota del examen final (variable dependiente) viene explicada por la nota obtenida en la evaluación continua, el número de pruebas realizadas para esta misma evaluación continua, y a su vez por cada uno de los formatos de las distintas pruebas (Díaz-Mora, García y Molina, 2016).

\section{Resultados}

\section{Análisis descriptivo}

En primer lugar, vamos a realizar un análisis descriptivo de la muestra destacando sus rasgos más significativos.

Si obtenemos las medias de las notas de la evaluación continua tanto por asignaturas y curso (Tabla 3), número de pruebas (Figura 1), como por tipo de prueba (Figura 2 y Tabla 4), podemos observar que esta nota media es baja en cualquiera de los casos (en ningún caso sube del 5.5 la nota media de la evaluación continua).

Analizando la nota de la evaluación continua en función del número de pruebas realizadas (Figura 1), las medias de notas obtenidas nos muestran que el incremento en el número de pruebas hace que se incremente la nota total de la evaluación continua.

Si analizamos la nota media de la evaluación continua en base a los distintos tipos de pruebas realizados (Figura 2), se puede observar que la mayor media se obtiene en la tarea, tanto presencial (un 8.16), como virtual (7.89).

Tabla 3

Nota media de evaluación continua por asignatura y curso académico

\begin{tabular}{lccc}
\hline Asignatura & Curso académico & Media & Desv. típ. \\
\hline \multirow{2}{*}{ 2210, Introducción a la contabilidad } & $2014-15$ & 2.90 & 1.700 \\
& Total & 2.90 & 1.700 \\
\hline \multirow{2}{*}{ 2344, Contabilidad Financiera I } & $2014-15$ & 4.61 & 1.688 \\
& $2015-16$ & 4.61 & 1.839 \\
& $2016-17$ & 4.49 & 1.966 \\
2351, Contabilidad Financiera II & Total & 4.58 & 1.842 \\
\hline \multirow{3}{*}{ 2357, Contabilidad Financiera Superior } & $2014-15$ & 3.29 & 2.344 \\
& $2015-16$ & 4.36 & 2.520 \\
& Total & 4.15 & 2.519 \\
\hline \multirow{2}{*}{ Total } & $2015-16$ & 3.41 & 2.207 \\
& $2016-17$ & 4.44 & 2.069 \\
& Total & 3.61 & 2.216 \\
\hline
\end{tabular}


En principio aquellas pruebas realizadas utilizando las nuevas tecnologías han obtenido una media mayor (Figura 2) en comparación con el resto.

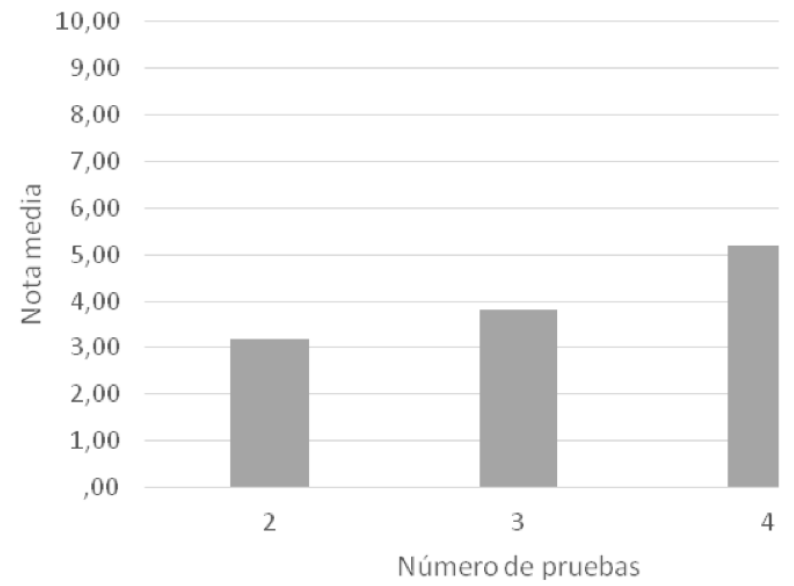

Figura 1. Media de notas de la evaluación continua por número de pruebas

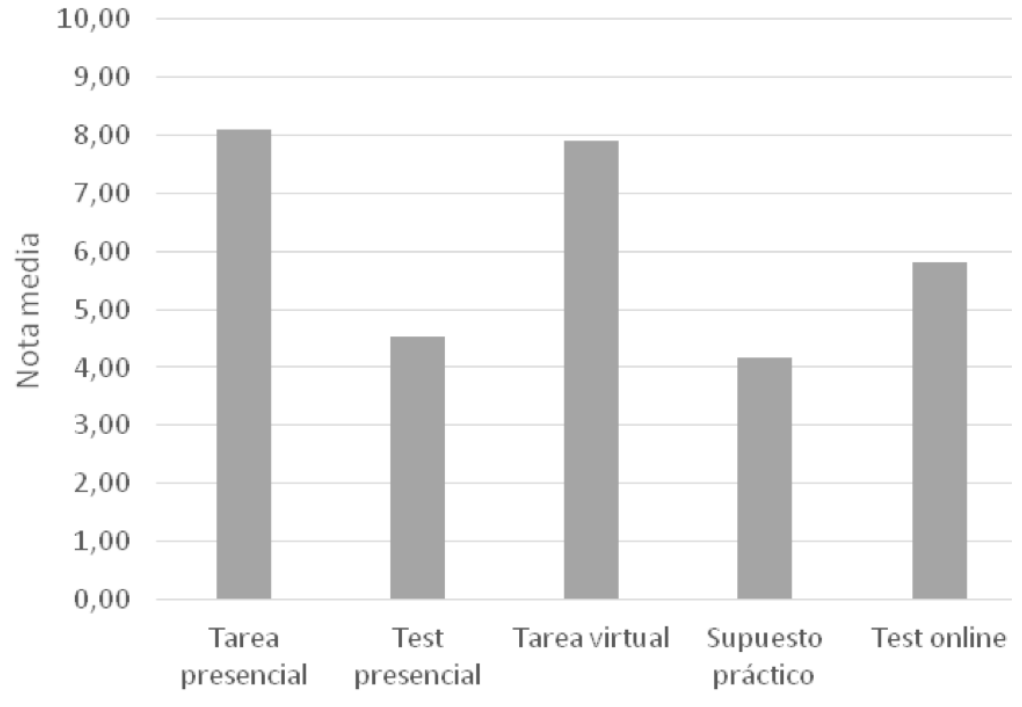

Tipo de prueba

Figura 2. Notas medias por tipo de pruebas de evaluación continua

Si analizamos las notas del examen final por tipo de prueba de evaluación continua realizada (Figura 3), se aprecia que la nota media del examen final se eleva a un 5.13 
cuando previamente había una tarea virtual en la evaluación continua, mientras que desciende casi un punto cuando había una tarea presencial.

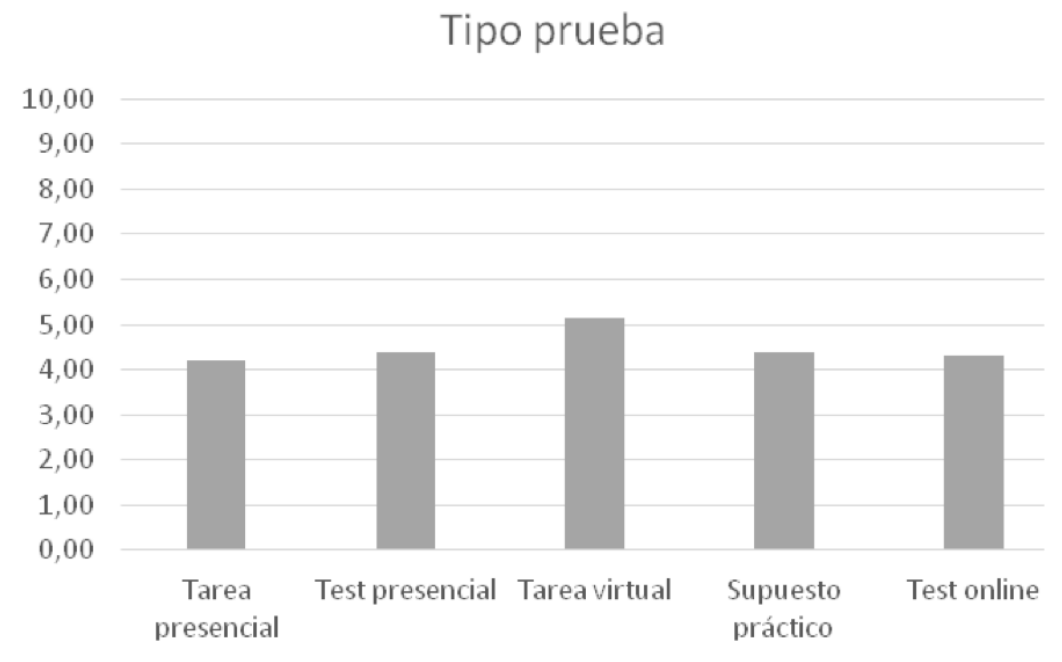

Figura 3. Media de nota del examen por tipo de pruebas de evaluación continua

\section{Análisis de tablas de contingencia y chi cuadrado}

Como se incluye en la Tabla 4, en todos los casos analizados se obtiene que existen influencias significativas al menos con una $p<.05$. Efectivamente hay un efecto del diseño de las pruebas de la evaluación continua en la calificación final de la asignatura, pudiéndola determinar, y no sólo a la nota del examen final, sino que también al hecho de que el alumno se presente al examen final y por lo tanto pueda optar a superar la asignatura.

Así, con respecto a los aprobados y suspensos en el examen y el número de pruebas parciales realizadas (Tabla 5) se puede observar que no existe un efecto sobre los mismos si van aumentando el número de pruebas parciales.

En la Tabla 6 podemos ver claramente que:

- la mayoría de los alumnos que no aprueban la evaluación continua no se presentan al examen final,

- la mayoría de los alumnos que aprueban la evaluación continua se presentan al examen final y además lo aprueban,

- que la mayoría de los alumnos que suspenden el examen final han suspendido previamente la evaluación continua, y

- que la mayoría de los alumnos que aprueban el examen final han aprobado la evaluación continua. 
Tabla 4

Chi cuadrado de los cruces de variables en las tablas de contingencia

\section{No presentados/Aprobados/suspensos} en el examen final

Número de pruebas parciales

Aprobados/Suspensos en la evaluación continua

Aprobados/Suspensos en la tarea presencial

Aprobados/Suspensos en la tarea virtual

Aprobados/Suspensos en el supuesto práctico

Aprobados/Suspensos en el test presencial

Aprobados/Suspensos en el test virtual

$$
\begin{aligned}
& \chi^{2}(4, \mathrm{~N}=1670)=12,92, \mathrm{p}=.012 * \\
& \chi^{2}(2, \mathrm{~N}=1670)=306,49, \mathrm{p}=0^{* *} \\
& \chi^{2}(2, \mathrm{~N}=1670)=50,27, \mathrm{p}=0 * * \\
& \chi^{2}(4, \mathrm{~N}=1670)=10,77, \mathrm{p}=.029^{*} \\
& \chi^{2}(4, \mathrm{~N}=1670)=458,43, \mathrm{p}=0^{* *} \\
& \chi^{2}(4, \mathrm{~N}=1670)=201,36, \mathrm{p}=0^{* *} \\
& \chi^{2}(4, \mathrm{~N}=1670)=147,20, \mathrm{p}=0^{* *}
\end{aligned}
$$

*Significativo al 95\%

**Significativo al 100\%

Tabla 5

Tabla de contingencia de aprobados/suspensos y número de pruebas parciales

\begin{tabular}{lccc}
\hline (no de casos) & \multicolumn{2}{c}{ Aprobados/suspensos en el examen } & Total \\
\cline { 2 - 3 } Número pruebas & Suspenso & Aprobado & 236 \\
\hline 2 & 121 & 115 & 225 \\
3 & 140 & 85 & 244 \\
4 & 126 & 118 & 705 \\
\hline
\end{tabular}

Tabla 6

Tabla de contingencia no presentados, suspensos y aprobados en el examen final y aprobados/suspensos evaluación continua

\begin{tabular}{lcccc}
\hline \multirow{2}{*}{ (no de casos) } & & \multicolumn{2}{c}{ Aprueba_eva_continua } & \multirow{2}{*}{ Total } \\
\cline { 3 - 4 } & & NO & SI & \\
\hline \multirow{3}{*}{ Examen final } & No presentado & 833 & 132 & 965 \\
& Suspenso & 281 & 106 & 387 \\
& Aprobado & 116 & 202 & 318 \\
\hline Total & & 1230 & 440 & 1670 \\
\hline
\end{tabular}


La Figura 4 muestra como la nota media en el examen final es muy superior para los alumnos que previamente habían aprobado la evaluación continua, frente a los que no la había superado.

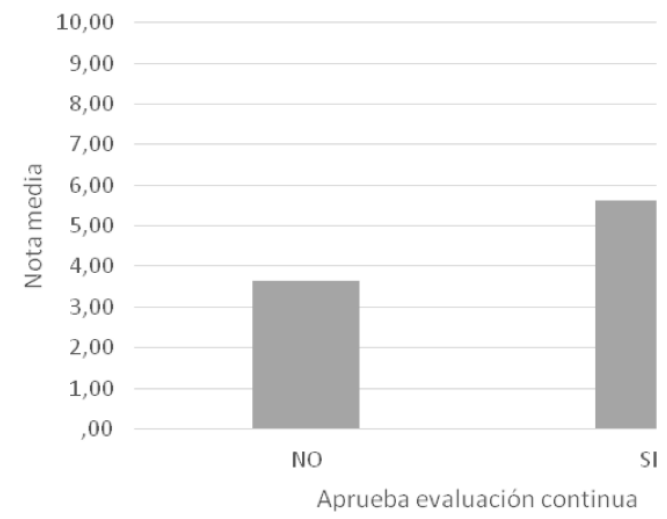

Figura 4. Media de nota del examen en función de aprobar o suspender la evaluación continua.

Tabla 7

Tabla de contingencia de no presentados, suspensos y aprobados en el examen final y aprobados/suspensos en formato tarea presencial

\begin{tabular}{ccccc}
\hline & & \multicolumn{3}{c}{ Aprueba tarea presencial } \\
\cline { 3 - 5 } & & NO & SI & Total \\
\hline \multirow{3}{*}{ Examen final } & No presentado & 511 & 226 & 737 \\
& Suspenso & 218 & 140 & 358 \\
& Aprobado & 119 & 191 & 310 \\
\hline \multirow{2}{*}{ Total } & 848 & 557 & 1405 \\
\hline
\end{tabular}

Siguiendo el mismo esquema anterior, se ha llevado a cabo un análisis de los resultados en el examen final, en función de los obtenidos en cada una de las distintas pruebas realizadas a lo largo de la evaluación continua. Si en primer lugar estudiamos el formato de tarea, ya sea presencial o virtual, se obtienen los siguientes resultados (Tabla 7):

- La mayoría de alumnos que no aprobaron la tarea presencial no se presentaron al examen final, y de los presentados, solo 1 de cada 3 consiguió aprobar.

- En cuanto a los que sí aprobaron la tarea presencial, la mayoría de alumnos se presentaron al examen final, y como era de esperar el éxito de este colectivo fue muy superior al de los que no habían superado esta prueba en la evaluación continua. 
- El cruce de las notas del examen final y de la tarea virtual no suministra resultados interesantes.

Al analizar el formato de prueba de supuesto práctico, incluido en la Tabla 8, y los resultados obtenidos en el examen final, debemos tener en cuenta que una de las partes del examen final es también un supuesto práctico y se realiza esta prueba intermedia como entrenamiento del mismo. Los resultados obtenidos refrendan que se entiende así por parte de los alumnos, puesto que:

- Los alumnos que no aprueban este formato de prueba no se presentan al examen final.

- Los alumnos que sí aprueban el supuesto práctico también aprueban mayoritariamente el examen final y así, al contrario.

Tabla 8

Tabla de contingencia de no presentados, suspensos y aprobados en el examen final y aprobados/suspensos en formato supuesto práctico de prueba parcial

\begin{tabular}{lcccc}
\hline & & \multicolumn{3}{c}{ Aprueba supuesto práctico } \\
\cline { 3 - 5 } & & NO & SI & Total \\
\hline \multirow{3}{*}{ Examen final } & No presentado & 353 & 114 & 467 \\
& Suspenso & 224 & 97 & 321 \\
& Aprobado & 115 & 189 & 304 \\
\hline & Total & 692 & 400 & 1092 \\
\hline
\end{tabular}

Tabla 9

Tabla de contingencia de no presentados, suspensos y aprobados en el examen final y aprobados/suspensos en formato tipo test presencial de prueba parcial

\begin{tabular}{|c|c|c|c|c|}
\hline & & \multicolumn{3}{|c|}{ Aprueba test presencial } \\
\hline & & NO & SI & Total \\
\hline \multirow{3}{*}{ Examen final } & No presentado & 511 & 226 & 737 \\
\hline & Suspenso & 218 & 140 & 358 \\
\hline & Aprobado & 119 & 191 & 310 \\
\hline \multicolumn{2}{|c|}{ Total } & 847 & 557 & 1405 \\
\hline
\end{tabular}

Para el formato de prueba tipo test podemos diferenciar entre modalidad presencial (Tabla 9) o modalidad online (indistintamente también la denominados virtual) (Tabla 10). En ambos casos el tipo test también constituye uno de los formatos de una de las partes del examen final. Los resultados obtenidos para el test presencial refrendan, al 
igual que la prueba con formato de supuesto práctico, que los alumnos lo consideran un indicador de su aprendizaje con respecto al examen final, pues según la Tabla 9:

- Los alumnos que no aprueban el test presencial tienden a no presentarse al examen final

- Los alumnos que no aprueban el test presencial y se presentan al examen final lo tienden a suspender, y así, al contrario.

Tabla 10

Tabla de contingencia de no presentados, suspensos y aprobados en el examen final y aprobados/suspensos en formato tipo test online de prueba parcial

\begin{tabular}{llccc}
\hline & & \multicolumn{3}{c}{ Aprueba test online } \\
\cline { 3 - 5 } & & NO & SI & Total \\
\hline \multirow{2}{*}{ Examen aprobado } & No presentado & 125 & 204 & 329 \\
& Suspenso & 110 & 115 & 225 \\
\hline Total & Aprobado & 43 & 150 & 193 \\
\hline
\end{tabular}

En cuando a la modalidad virtual (Tabla 10) el número de alumnos que se presenta a esta prueba es más reducido, ya que normalmente es la prueba de evaluación continua que se realiza al final de todo el cuatrimestre, cuando se supone que los alumnos ya han decidido si continuar o no con el aprendizaje de la asignatura para ir al examen final, además de que los aprobados se elevan considerablemente con respecto al test presencial.

\section{Análisis explicativo a través de regresiones}

En el modelo 1 de regresión que planteamos, incluimos como variable dependiente la nota del examen final, y como variables explicativas la nota obtenida en la evaluación continua, el número de pruebas realizadas para esta misma evaluación continua, y a su vez cada uno de los formatos de las distintas pruebas. En línea con lo que se había obtenido en el análisis estadístico previo, los coeficientes significativos más explicativos de la nota del examen final, son los del supuesto práctico y el test presencial. Mientras que el resto de tipos de pruebas de evaluación continua apenas tienen carácter explicativo sobre la nota del examen final, o incluso no son significativas, como sucede con la tarea presencial (Tabla 11).

En el modelo 2 realizamos la regresión incluyendo como variable dependiente la nota del examen final y como variables independientes las notas de los cinco tipos de pruebas de evaluación continua, consiguiendo un aumento en el grado de bondad del ajuste (con una mayor $\mathrm{R}^{2}$ en el modelo 2 de la tabla 11) y obtenemos que la relación 
es estadísticamente significativa, por lo tanto, de nuevo se refrenda nuestra $\mathrm{H}_{3^{\prime}} \mathrm{y}$ a su vez, que otra vez los resultados de las pruebas tipo supuesto práctico y test presencial son los que más influyen sobre la nota del examen final.

Tabla 11

Estimación de los modelos de regresión

\begin{tabular}{|c|c|c|c|c|}
\hline \multirow[b]{2}{*}{$N=1670$} & \multicolumn{2}{|c|}{ Modelo 1} & \multicolumn{2}{|c|}{ Modelo 2} \\
\hline & $\beta$ (ET) & $\mathrm{p}$ & $\beta$ (ET) & $\mathbf{P}$ \\
\hline Constante & $1.744(0.208)$ & .000 & $2.110(0.185)$ & .000 \\
\hline $\begin{array}{l}\text { Nota de evaluación } \\
\text { continua }\end{array}$ & $0.582(0.040)$ & .000 & - & - \\
\hline Número pruebas & $0.009(0.105)$ & .931 & - & - \\
\hline Tarea presencial & $-0.013(0.035)$ & .718 & $-0.026(0.032)$ & .409 \\
\hline Tarea virtual & $0.088(0.028)$ & .002 & $0.065(0.027)$ & .016 \\
\hline Test online & $0.122(0.025)$ & .000 & $0.059(0.024)$ & .017 \\
\hline Test presencial & $0.349(0.035)$ & .000 & $0.18(0.037)$ & .000 \\
\hline Supuesto práctico & $0.356(0.027)$ & .000 & $0.304(0.028)$ & .000 \\
\hline $\mathrm{R}^{2}$ ajustado (\%) & \multicolumn{2}{|c|}{24.6} & \multicolumn{2}{|c|}{25.8} \\
\hline Modelo & \multicolumn{2}{|c|}{$\mathrm{F}(7)=43.9, \mathrm{p}=.000$} & \multicolumn{2}{|c|}{$F(5)=49.87, p=.000$} \\
\hline
\end{tabular}

\section{Conclusiones}

Tras el estudio realizado, podemos afirmar que la evaluación continua favorece el aprendizaje en las asignaturas de contabilidad financiera, lo que se refleja en la nota del examen final, tal y como comprueban también otros autores como Cano (2011), Fraile et al. (2013), López-Pastor (2011), y Moro-Egido (2016), o que los métodos de enseñanza-aprendizaje tienen efecto en la nota media como obtienen Coll-Serrano et al. (2018). En nuestro caso efectivamente la nota de la evaluación continua explica en una parte importante (el coeficiente obtenido en el modelo de regresión es 0.582 ) la nota del examen final, por lo que claramente si el alumno ha aprovechado la evaluación continua para su aprendizaje esto repercutirá positivamente en su nota (Se comprueba la hipótesis $\mathrm{H}_{1}$ ). En cambio, el número de pruebas que se realizan en la evaluación continua en principio no resulta significativo, y por lo tanto, no podemos aceptar nuestra segunda hipótesis $\mathrm{H}_{2}$. Con respecto a $\mathrm{H}_{3}$ que se refería al tipo de prueba, sí que afecta a la nota del examen final, pero claramente hay que distinguir entre los cinco formatos de pruebas empleados en nuestro estudio e incluidos en la muestra estudiada. En aquellas pruebas en las que se utilizan las nuevas tecnologías, se contribuye en mayor manera a elevar la nota de la evaluación continua, de manera que en aquellos casos en los que se les ha realizado a los alumnos una prueba tipo test, la 
nota ha sido superior cuando ésta se ha realizado de manera virtual a los casos en los que se llevó a cabo la prueba presencialmente, tal y como comprueban Inda, Alvárez y Alvárez (2008), cuando aseguran que las metodologías más innovadoras tienen unos efectos más positivos sobre la nota. Aunque en teoría los resultados obtenidos están de acuerdo con los estudios previos, en la práctica se debe profundizar en el diseño del tipo de pruebas que se realizan en la evaluación continua. Ya que si bien se aboga por distintos formatos que vayan cambiando curso a curso (Crisp, 2007), nuestros resultados plantean que el formato usado en las distintas pruebas que constituyen la evaluación continua puede determinar la nota final. Principalmente se pone de manifiesto una relación positiva entre aquellas pruebas que son una réplica del formato del examen final y la nota conseguida en el mismo, ya que sirven claramente al alumno como entrenamiento para este aprendizaje y como indicador para saber si está preparado para presentarse y superar el examen.

El análisis de los resultados obtenidos nos lleva a concluir que, aunque efectivamente parece que la evaluación continua tiene efectos positivos en la nota final del examen, y así en el aprendizaje, es fundamental el diseño de las pruebas que la configuran, porque a su vez puede determinar estos efectos. De manera que se plantea como futura línea de investigación, el seguir profundizando en el análisis de las notas obtenidas de la evaluación continua y las notas finales, a través del estudio de muestras mayores y series de datos en las que se incluya la mayor tipología posible de formatos de pruebas de evaluación continua. Además, pretendemos estudiar, en qué medida ayuda al aspecto formativo de esta evaluación, el uso de juegos como pruebas de evaluación continua, y si tienen efecto en la nota final, utilizando para ello aplicaciones (como kahoot), que nos brindan actualmente las nuevas tecnologías. Otra línea de estudio en la que seguir trabajando, es el análisis del tipo de pruebas de evaluación continua para alumnos con necesidades educativas especiales en la universidad, o las diferencias obtenidas en las notas de estas pruebas, dependiendo del género de los estudiantes.

\section{Referencias}

Alcaraz, N., Fernández, M. y Sola, M. (2012). La voz del alumnado en los procesos de evaluación docente universitaria. Revista Iberoamericana de Evaluación Educativa, 5(2), 26-39

Barrientos Hernán, E. J. y López Pastor, V. M. (2017). Evaluación orientada al aprendizaje en educación superior: últimas tendencias. Infancia, Educación y Aprendizaje, 3(2), 582-587.

Bennet, R. E. (2011). Formative assessment: a critical review. Assessment in education: principles, policy and practice, 18(1), 5-25.

Birenbaum, M., Earl, L., Heritage, M., Klenowski, V., Looney, A., Smith, K., Timperley, H., Volante, L. y Wyatt-Smith, C. (2015). International trends in the implementation of assessment for learning: Implications for policy and practice. Policy Futures in Education, 13(1), 117-140. doi: 10.1177/1478210314566733.

Black, P. (2015). Formative assessment- an optimistic but incomplete vision. Assessment in Education: Principles, Policy E Practice, 22(1), 161-177. doi: 10.1080/0969594X.2014.999643. 
Black, P. y William, D. (1998). Assessment and Classroom Learning. Assessment in Education: Principles, Policy and Practice, 5(1), 7-74. doi: 10.1080/0969595980050102.

Camacho Miñano, M. M., Urquía Grande, E., Pascual Ezama, D. y Rivero Menéndez, M. J. (2016). Recursos multimedia para el aprendizaje de contabilidad financiera en los grados bilingües. Educación XXI, 19(1). doi: 10.5944/educXX1.13941.

Cano, M. D. (2011). Students' involvement in continuous assessment methodologies: A case study for a distributed information systems course. IEEE Transactions on Education, 54(3), 442-451. doi:10.1109/TE.2010.2073708.

Carless, D. (2007). Learning-oriented assessment: conceptual bases and practical implications. Innovations in Education and Teaching International, 44(1), 57-66.

Carless, D. (2015). Exploring learning-oriented assessment processes. Higher Education, 69(6), 963-976.

Castejón, F. J. y Santos, M. L. (2011). Percepciones y dificultades en el empleo de metodologías participativas y evaluación formativa en el Grado de Ciencias de la Actividad Física. Revista Electrónica Interuniversitaria de Formación del Profesorado (REIFOP), 14(4), 117-126.

Coll-Serrano, V., Pardo-García, C. y Pérez, P. J. (2018). Teaching-learning methods and their effect on profesional development and the development of graduates' competencies/ Métodos de enseñanza-aprendizaje y su efecto en el desarrollo profesional y competencial de los egresados. Cultura y Educación, 30(3). doi: 10.1080/11356405.2018.1494773.

Crisp, B. R. (2007). Is it worth the effort? How feedback influences students' subsequent submission of assessable work. Assessment and Evaluation in Higher Education, 32(5), 571-581.

Crooks, T. J. (1988). The impact of classroom evaluation practices on students. Review of Educational Research, 58(4), 438-481.

Díaz-Mora, C., García, J. A. y Molina, A. (2016). What is the key to academic success? An analysis of the relationship between time use and student performance. Cultura y Educación, 28(1), 157-195. doi: 10.1080/11356405.2015.1130294.

Dowell, L. Mc., Sambell, K., Bazin, V., Penlington, R., Wakelin, D., Wickes, H. y Smailes, J. (2007). Assessment design for learner responsibility. Recuperado de http://www. reap.ac.uk/reap/reap07/Portals/2/CSL/feast\%20of\%20case\%20studies/Assessment_ for_learning_current_practice_exemplars_from_CETL.pdf.

Dunn, K. E. y Mulvenon, S. W. (2009). A critical review of research on formative assessments: The limited scientific evidence of the impact of formative assessments in education. Practical Assessment and Research and Evaluation, 14(7), 1-11.

Escámez Sánchez, J. (2016). La excelencia en el profesor universitario. Revista española de pedagogía, (254), 11-27.

Fernández, S. (2017). Evaluación y aprendizaje. Revista de didáctica español como lengua extranjera, 24, 1-43.

Fernández-Ponce, C., Arbulo Echevarría, M. M., Muñoz-Miranda, P., Aguado, E. y García-Cozar, F. (2017). Análisis de la evaluación continua en el contexto del EEES. Docencia e Investigación, 27, 81-100.

Flores, M. A., Veiga Simao, A. M., Barros, A. y Pereira, D. (2015). Perceptions of effectiveness, fairness and feedback of assessment methods: a study in higher educa- 
tion. Studies in Higher Education 40Studies in Higher Education, 40(9), 1523-1534. doi: 10.1080/03075079.2014.881348.

Fraile, A., López, V. M., Castejón, F. J. y Romero, R. (2013). La evaluación formativa en docencia universitaria y el rendimiento académico del alumnado. Aula Abierta, (41), 23-34.

Fuchs, L. S. y Fuchs, D. (1986). Effects of systematic formative evaluation: a metaanalysis. Exceptional children, (53), 199-208.

Gámiz Sánchez, V. y Gallego Arrufat, M. J. (2016). Modelo de análisis de metodologías didácticas semipresenciales en educación superior. Educación XXI, 19(1), 39-61. doi: 10.5944/educXXI.13946.

Gargallo López, B., Morera Bertomeu, I., Iborra Chornet, S., Climent Olmedo, M. J., Navalón Oltra, S. y García Félix, E. (2014). Metodología centrada en el aprendizaje. $\mathrm{Su}$ impacto en las estrategias de aprendizaje y en el rendimiento académico de los estudiantes universitarios. Revista española de pedagogía, (259), 415-435.

Gibbs, G. (2006). Why assessment is changing. En C. Bryan y K. Klegg (Eds.) Innovative Assessment in Higher Education (pp.11-22). Abingdon: Routledge

Gibbs, G. y Simpson, C. (2004). Conditions under which assessment supports students' learning. Learning and teaching in higher education, 1, 3-31.

Gil Flores, J. y García Jiménez, E. (2014). Evaluación formativa y resultados de aprendizaje en los centros que imparten Educación Secundaria Obligatoria. Revista Española de pedagogía, (259), 437-455.

Heitink, M. C., Van der Kleij, F. M., Veldkamp, B. P., Schildkamp, K. y Kippers, W. B. (2016). A systematic review of prerequisites for implementing assessment for learning in classroom practice. Educational Research Review, 17, 50-62. doi: 10.1016/j. edurev.2015.12.002.

Hernández, R. (2012). Does continuous assessment in higher education support student learning? Higher Education, (64), 489-502. doi: 10.1007/s10734-012-9506-7.

Inda Caro, M., Âlvarez González, S. y Álvarez Rubio, R. (2008). Métodos de evaluación en la enseñanza superior. Revista de Investigación Educativa, 26, 539-552.

Isaksson, S. (2008). Assess as you go: the effect of continuous assessment on student learning during a short course in archaeology. Assessment and Evaluation in Higher Education, 33(1), 1-7. doi: 10.1080/02602930601122498).

Jareño Cebrián, F. y López García, R. (2015). Actividades de evaluación continua- correlación con la calificación de la prueba final y efecto sobre la calificación final. Evidencia en Administración y Dirección de Empresas. Revista Complutense de Educación, 26(2), 241-254.

Kluger, A. N. y DeNisi, D. A. (1996). The effects of feedback interventions on performance: A historical review, a meta-analysis, and a preliminary feedback intervention theory, Psychological Bulletin, 119(2), 254-284.

López-Pastor, V. M. (2011). El papel de la evaluación formativa en la evaluación por competencias: Aportaciones de la red de evaluación formativa y compartida en docencia universitaria. Revista de Docencia Universitaria, 9(1), 159-173.

Martínez Clares, P., Pérez Cusó, J. y Martínez Juárez, M. (2016). Las TICs y el entorno virtual para la tutoría universitaria. Educación XXI, 19(1), 287-310. doi: 10.5944/ educXXI.13942. 
Monroy, F. y Hernández Pina, F. (2014). Factores que influyen en los enfoques de aprendizaje universitario. Una revisión sistemática. Educación XXI, 17(2), 105-124. doi: 10.5944/educxx1.17.2.11481.

Moreno-Murcia, J. A., Aracil, A. y Reina, R. (2014). La cesión de responsabilidad en la evaluación: una estrategia adaptada al Espacio Europeo de Educación Superior. Educación XXI, 17(1), 183-200. doi: 10.5944/educxx1.17.1.10710.

Moro-Egido, A. I. (2016). An assessment of evaluation techniques in higher education: the experience of macroeconomics. Cultura y Educación, 28(4), 826-862. doi: 10.1080/11356405.2016.1230294.

Navaridas Nalda, F., Jiménez Trends, M. A. y Fernández Ortiz, R. (2016). El aprendizaje de competencias en la Universidad: expectativas predictivas y niveles de confirmación de los estudiantes. Revista española de pedagogía, (264), 337-356.

Palacios Picos, A. (2004). El crédito europeo como motor de cambio en la configuración del Espacio Europeo de la Educación Superior. Revista Interuniversitaria de Formación del Profesorado, 18(3), 197-205.

Parkes, K. A. (2010). Performance Assessment: Lessons from Performers. International Journal of Teaching and Learning in Higher Education, 22(1), 98-106.

Pascual Ezama, D., Camacho Miñano, M. M., Urquía Grande, E. y Müller, A. (2011). ¿Son los nuevos criterios de evaluación en el marco del EEES adecuados para valorar el rendimiento académico de los alumnos? Experiencia en Contabilidad Financiera. Revista de Educación en Contabilidad, Finanzas y Administración de Empresas, (2), 67-83.

Pereira, D., Niklasson, L. y Flores, M. A. (2017). Students' perceptions of assessment: a comparative analysis between Portugal and Sweden. Higher Education, 73, 153-173.

Poza-Lujan, J. L., Calafate, C. T., Posadas-Yague, J. L. y Cano, J. C. (2016). Assessing the Impact of Continuous Evaluation Strategies: Tradeoff between Student Performance and Instructor Effort. IEEE Transactions on Education, 59(1), 17-23. doi: 10.1109/TE.2015.2418740.

Rodríguez-Gómez, G., Quesada-Serra, V. y Ibarra-Sáiz, M. S. (2016). Learning-oriented e-assessment: the effects of a training and guidance programme on lecturers' perceptions. Assessment $\mathcal{E}$ Evaluation in Higher Education, 41(1), 35-52. doi: 10.1080/02602938.2014.979132.

San Martín Gutiérrez, S., Jiménez Torres, N. y Jerónimo Sánchez-Beato, E. (2016). La evaluación del alumno universitario en el Espacio Europeo de Educación Superior. Aula Abierta, 44, 7-14. doi: 10.1016/j.aula.2015.03.003.

Sánchez-Martín, M. P., Pascual-Ezama, D. y Delgado-Jalón, M. L. (2017). Estudiantes mejor informados: mejores resultados académicos. Revista de Contabilidad-Spanish Accounting Review, 20(1), 47-54.

Shute, V.J. (2008). Focus on formative feedback. Review of educational research, 7(1), 153-189.

Trigueros Cervantes, C., Rivera García, E. y De la Torre Navarro, E. (2012). La evaluación en el aula universitaria: del examen tradicional a la autoevaluación. Revista Internacional de Medicina y Ciencias de la Actividad Física y el Deporte, 12(47), 473-491.

Trotter, E. (2006). Student perceptions of continuous summative assessment. Assessment and Evaluation in Higher Education, 31(5), 505-521. 
Vallet-Bellmunt, T., Rivera-Torres, P. y Vallet-Bellmunt, I. (2017). Aprendizaje cooperativo, aprendizaje percibido y rendimiento académico en la enseñanza del marketing. Educación XXI, 20(1), 277-297. doi: 10.5944/educXX1.11408.

Yorke, M. (2003). Formative Assessment in higher education: Moves Howard theory and the enhancement of pedagogic practice. Higher education, 45(4), 477-501.

Fecha de recepción: 1 de mayo de 2018.

Fecha de revisión: 21 de mayo de 2018.

Fecha de aceptación: 21 de enero de 2019. 
